\title{
Exode et domesticité à Bogotá (Colombie)
}

\author{
Félicie Drouilleau
}

Doctorante au Laboratoire LISST-CAS

Les travaux sociologiques, les statistiques nationales, la littérature [Quintero de Molano, 1981, p. 2 ; Martínez Gómez, 2006, p. 169 et p. 179 ; Osorio Lizarazo, 2008 (1952), p. 9-22] et tout simplement l'imaginaire collectif ont mis en évidence la figure «traditionnelle » d'une migration, celle des petites filles ou des jeunes filles, recrutées comme domestiques à Bogotá ou dans des villes intermédiaires ${ }^{1}$. Le personnage de cette jeune migrante, paysanne, se retrouve de manière particulièrement pathétique dans le roman de José Antonio Osorio Lizarazo, El día del odio [2008 (1952)]. L'ouvrage, qui narre la genèse du Bogotázo ${ }^{2}$, commence par le récit de la mise au travail de la jeune Transito, petite paysanne naïve de Boyacá ${ }^{3}$, que sa mère présente sur le marché pour la placer comme domestique dans la capitale; une fois sa fille employée, la mère vient prendre l'argent du travail de Transito sans se soucier de son bien être. Métisse indienne, Transito est une figure représentative du type d'immigration rurale cundiboyacens $^{4}$, dans la capitale colombienne durant la première moitié du vingtième siècle [Cardona Gutierrez, 1970, p. 164 ; Fierro, 1973, p. 18-22]. Cette migration, qui n'est pas à proprement parler « indienne », provient d'une région où le métissage laisse deviner une forte ascendance indienne et andine. L'usage, tout au long du roman, du terme péjoratif «india» rappelle que si on peut parler à son propos de métissage, Transito demeure finalement une « indienne », et ses yeux clairs ne changent rien à l'affaire.

Protagoniste tragique d'un événement qui modifiera profondément l'histoire du pays (l'assassinat du chef de file libéral Jorge Eliécer Gaitán le 9 avril 1948), Transito ne connaîtra pas ses suites, la guerre civile anarchique, communément nommée «La Violence», opposant les tenants des deux principaux partis politiques colombiens. Ces affrontements entraîneront la mort de plus de 200000 personnes [Agier et Hoffmann, 1999, p. 108] et l'exil de près de 400000 familles paysannes [Osorio Pérez, 2003, p. 38]. Ils modifieront également en profondeur la nature des migrations internes dans le pays. Comme l'indique Gonzalo Sánchez dans son ouvrage Guerre et 
politique en Colombie « (...) l'espace occupé par les conflits [se définissait à cette époque] en terme de persécution, de diaspora et de fuite, dans des directions multiples : de la campagne vers les villes, du village vers la métropole, du centre du pays vers les terres lointaines de colonisation, de la Colombie vers les pays voisins. »[1998, p. 36]. Cette période de profonde confusion a duré jusqu'au début des années 1960 et a laissé place à un important développement industriel urbain favorisé par la pacification du pays et une économie basée sur la substitution aux importations [Meertens, 2000, p. 227]. Les migrations se sont alors concentrées vers les plus grandes métropoles de la Colombie, en particulier vers sa capitale Bogotá, provoquant un phénomène d'urbanisation massive. La mécanisation agricole, la déstructuration de la famille paysanne, la concentration dans les villes des principales avancées sociales ainsi que le fort taux de croissance démographique des campagnes peuvent expliquer cet «exode rural » d'une ampleur sans précédent [Martínez Gómez, 2006, p. 31]. Les migrations des années 1960-1970 concernent une majorité de jeunes femmes seules et sans enfants qui arrivent en ville à la recherche d'un emploi - souvent le travail domestique interno $^{5}$ - et d'éducation. Dans la capitale, les migrants ne proviennent plus simplement de Cundinamarca et Boyacá mais également d'autres départements comme le Tolima, Santander, Caldas et Valle [Fierro, 1973, p. 21].

A partir du début des années 1980, cette migration rurale vers la ville se fait moins pressante. La croissance chaotique des centres urbains ainsi que la détérioration des conditions de vie dans les quartiers périphériques où se sont concentrés les nouveaux migrants dissuadent d'une venue en ville. Parallèlement au ralentissement de ces migrations, une nouvelle phase de violence voit le jour. L'expansion du narco-trafic, les nouvelles offensives de la guérilla et l'apparition des groupes paramilitaires affectent tout particulièrement les zones rurales. Cette situation entraîne les déplacements forcés de paysans fuyant les massacres, les assauts des différents groupes armés, les enlèvements et de multiples formes de persécutions. Ces migrants ne cherchent plus essentiellement du travail ou un accès à l'éducation mais un refuge et la sécurité [Martínez Gómez, 2006, p. 33]. Ils se dirigent vers les villes les plus proches ou les grandes métropoles où ils viendront grossir les ceintures de misère, déjà saturés par les précédents flux migratoires. Le terme « déplacement forcé », forgé par les organisations non gouvernementales, apparaît dans les discours officiels en 1995 [Meertens, 1999, p. 414] en même temps que les premières estimations chiffrées. Des études spécifiquement dédiées à cette population se font jour. On apprend ainsi que les femmes sont majoritaires. Il s'agit de mères de famille qui doivent lutter pour la survie du foyer, parfois seules, 
car leurs maris ont été assassinés ou recrutés par un groupe armé. Elles trouvent alors du travail comme employées domestiques journalières ou dans la vente ambulante et leur participation économique au sein de la famille devient essentielle [Meertens, 1999, p. 418 ; Meertens, 2001, p. 123]. A Bogotá, les familles déplacées proviennent de l'ensemble du territoire : Meta, Antioquia, Bolivar, Caquetá, Tolima, Boyacá, Casanare, Chocó, etc. [Meertens, 1999, p. 411]. Au tournant du siècle, la guerre s'intensifie ainsi que les déplacements massifs de populations, qui affectent aujourd'hui presque quatre millions de personnes [FAMIG-CODHES, 2007, p. 43]. En 1999, Martha Nubía Bello et Claudia Mosquera pouvaient ainsi encore affirmer : «le fait que [les personnes déplacées] n'arrivent pas massivement [à Bogotá] et que, donc, [elles] ne forment pas un collectif différencié, «un autre», empêche les réactions de signalement ou d'agression car la communauté ne se sent pas menacée $»^{6}[1999$, p. 466]. Dix ans plus tard, la situation est toute autre. Alors qu'en 1996, vingt-sept personnes déplacées arrivaient en moyenne chaque jour à Bogotá [Meertens, 1999, p. 410], aujourd'hui elles sont près d'une centaine [FAMIG-CODHES, 2007, p. 43]. Les médias, les universitaires, les organisations gouvernementales et les politiques publiques qui avaient contribué à faire émerger la catégorie de « déplacé » dans le courant des années 1990 [Osorio Pérez, 2002, p. 85] ont continué à sensibiliser le grand public sur ce phénomène, renforçant d'une certaine manière la réalité de cette catégorie. L' «autre » n'est ainsi plus aujourd'hui, à Bogotá, la jeune paysanne de Boyacá venue chercher du travail comme bonne à demeure, mais bien le groupe hétérogène [Agier et Hoffmann, 1999, p. 114] des personnes déplacées, qui amènent avec elles le soupçon d'une transmission à la ville des horreurs de la guerre.

Aujourd'hui, le service domestique regroupe presque un million de personne en Colombie dont un peu plus de 200000 dans la capitale, ce qui représente 15\% des femmes actives. A Bogotá, entre 1996 et 2004, le nombre de personnes employées dans cette branche d'activité a connu une forte hausse [Cárdenas et Harker, 2006, p. 6]. Cette augmentation s'est accompagnée d'une nette diminution des salaires pour les employées domestiques externas. Ces femmes travaillent aujourd'hui plus por días qu'interna et sont souvent mères de famille [Cárdenas et Harker, 2006]. Il est malaisé de saisir l'importance du déplacement forcé dans ce nouveau visage de l'emploi domestique. En effet, les données chiffrées manquent ou sont souvent partielles ${ }^{7}$. Pour mieux comprendre les spécificités du parcours migratoire et de l'insertion dans le service domestique des femmes déplacées, nous avons choisi de les confronter à ceux des jeunes filles ou petites filles qui ont migré et commencé à travailler comme petites bonnes dans les années 1960-1970. Il peut être 
intéressant en effet de comparer les deux vagues de migrantes à Bogotá : celles qui sont arrivées jeunes, voire enfants, en plein exode rural dans les années 1960-1970, fortes d'un désir d'ascension sociale et d'une vie meilleure dans la capitale, et la fuite désespérée de femmes, plus âgées, mères de famille, luttant pour leur survie quotidienne dans une ville où la délinquance et la misère gagnent chaque jour plus de terrain. Comment ces deux générations d'employées domestiques ont-elles été reçues par les familles employeuses ? D'un pays en paix, où les questions d'insécurité étaient temporairement - devenues marginales, à une Colombie déchirée par le conflit armé, comment s'organise l'entrée dans les foyers des employées domestiques?

\section{Emploi domestique et mise au travail des petites filles ${ }^{8}(1960-1970)$}

Si l'on en croit les témoignages que nous avons recueillis ${ }^{9}$, l'entrée dans une famille d'employeur était, durant les années 1960-1970, en grande partie gérée par les liens familiaux. Il pouvait s'agir du placement chez un oncle ou une tante en cas de décès d'un des parents, comme de $l^{\prime}$ «envoi » en ville de ses enfants chez des membres de la famille pour qu'ils puissent continuer à étudier. Très souvent, la contrepartie à une telle prise en charge éducative était le travail comme petits domestiques des enfants alors accueillis. Ce travail familial était généralement gratuit. Ainsi, le père de María, née à la fin des années 1950, est mort quand elle avait 13 ans. Elle raconte : «Mon oncle a dit que d'accord, il prendrait l'enfant le plus jeune. Et ma mère s'est exclamée : "Et bien maintenant il faut que je les répartisse comme des petits poulets ! ». Alors mes deux soeurs ont eu de la chance car elles sont allées chez des tantes qui n'avaient pas d'enfant et elles ont pu passer le baccalauréat. Alors que moi non. (...) Mon enfance ça a été : je me levais à quatre heures du matin pour moudre le maïs (....) je faisais les arepas ${ }^{10}$, la soupe, le chocolat, il fallait que j'emmène les deux garçons à l'école (...) Ma tante allait au travail et mon oncle aussi. Et moi je restais toute seule et je faisais le ménage » María est ainsi restée jusqu'à ses 30 ans chez sa tante et a pu finalement faire des études. Aujourd'hui, elle a une cinquantaine d'années et travaille toujours comme employée domestique chez des membres de sa famille. Toutefois, elle reçoit une rétribution pour ses services. On pourra noter que ce travail domestique familial s'effectue dans des quartiers plutôt populaires de Bogotá comme Kennedy ou Tunal. De la même manière, Blanca qui a commencé à travailler dans les années 1970 comme petite bonne chez sa tante pour payer sa scolarisation à Bogotá, a finalement continué dans ce type d'emploi, dans le cercle familial, puisqu'elle va dorénavant faire le ménage chez un de ses neveux, mais aussi chez des 
connaissances. Les frères et soeurs de Blanca, qui ont le même parcours, ont su, pour leur part, profiter de l'éducation qui leur a été donnée pour s'employer dans une autre branche socioprofessionnelle, parfois mieux rémunérée, comme le secrétariat ou la comptabilité. Ce travail familial, en échange d'une prise en charge éducative, semble exister encore de nos jours. Au cours de notre enquête dans la capitale colombienne, nous avons fait la connaissance de Cristina, venue de la côte Atlantique à Bogotá chez sa tante pour continuer ses études supérieures. Quelques mois après, lasse de n'être occupée qu'à des tâches domestiques, elle a décidé de rentrer chez elle et a monté un commerce sur la côte ${ }^{11}$.

L'insertion dans le travail domestique, qui peut perdurer ou non avec l'âge, s'effectue ici dans le strict cadre des relations de parenté. La confiance en l'employée vient tout naturellement de son statut de membre de la famille et le service domestique ne représente pas, dans ces circonstances, l'entrée d'une personne étrangère dans le foyer, mais l'utilisation des forces familiales en vue d'une certaine solidarité éducative ou, selon les cas, d'une exploitation par la parenté.

Un autre type de placement familial commun dans les années 1960-1970 est celui de la mise au travail des petites filles dans des villages ou maisons voisines. Elles n'étaient parfois pas emmenées directement à Bogotá, mais placées par leurs parents chez des voisins ou connaissances pour faire le ménage et garder des enfants. Ce type de mise au travail était généralement compris par ces enfants comme une forme d'exploitation et non comme une prise en charge à visée éducative. Ils recevaient un salaire, directement capté par les parents. Angelica, 49 ans aujourd'hui, s'est enfuie de la maison familiale quand elle avait une douzaine d'années. Sa mère travaillait pour des hacendados ${ }^{12}$ espagnols dans une ferme du Cauca. D'origine indienne, elle était amante de ce propriétaire terrien, par ailleurs chef de famille et incorrigible coureur de jupons. Elle a eu huit enfants dont trois seulement sont restés en vie. Mère seule, elle battait jusqu'au sang Angelica. Un jour, Angelica est partie, mais quelques mois plus tard, elle a été retrouvée par sa mère qui l'a alors placée dans une maison voisine comme petite domestique : «Ma maman est venue et elle m'a sortie de là. Et elle m'a laissée dans un endroit peu recommandable. (...) Mais c'était elle qui en profitait : elle venait et prenait l'argent sur mon dos, sans se soucier de savoir si j'étais bien, si j'avais de bons habits, des chaussures, non rien! ». De la même manière, Andrea, âgée aujourd'hui de 55 ans, est allée travailler très jeune chez une voisine du village. Ses parents tenaient un salon de coiffure en Boyacá. Ils ont eu une dizaine d'enfants, dont Andrea, qui se situe au milieu de la fratrie : 
«Quand j'avais neuf ans je suis partie de la maison. C'était parce que ma maman ne faisait pas attention à nous. Il y avait une femme qui vivait dans la maison d'à côté. Elle avait un enfant et elle voulait que je le garde. (...) Elle a parlé avec ma mère et lui a demandé de me laisser là-bas. Elle disait qu'elle allait me payer. (...) Mais ma mère venait et prenait tout ce que je gagnais ». Andrea est restée quelques années chez ces employeurs qui l'ont finalement adoptée. Victime d'agressions sexuelles de la part du patron ${ }^{13}$, elle a fui vers Bogotá pour y travailler comme employée domestique.

Ces petites filles commençaient à travailler comme bonnes à demeure en fonction d'un contrat tacite passé entre les parents et des voisins ou connaissances. Les liens de proximité remplaçaient, dans ce cas, les liens familiaux pour «faire rentrer » l'enfant dans une maison et établir une certaine confiance dans l'employée. On pourra noter que ces deux cas, l'emploi domestique de petites filles dans le strict cadre de la parenté, et la mise au travail d'enfants dans le voisinage, se rapprochent des situations décrites par Mélanie Jacquemin, de «petites nièces » et d'«enfants loués » à Abidjan (2009, 2007). Comme dans la capitale économique ivoirienne, en Colombie, l'entrée dans le service domestique se faisait, dans les années 1960-1970, en fonction de dynamiques familiales ou de proximité, qui pouvaient prendre la forme d'un apprentissage par le travail ou bien d'une exploitation éhontée.

D'autres petites filles ou jeunes filles, en revanche, pouvaient s'employer dans une maison à la suite d'une démarche personnelle. La fuite était un phénomène assez courant pour expliquer l'arrivée de ces enfants ou jeunes femmes à Bogotá. «Je me suis enfuie de la maison» ou «j'ai décidé de partir» sont des expressions que nous avons souvent retrouvées dans les entretiens. Il s'agissait alors du foyer familial comme de la maison où l'enfant avait été placée par ses parents. Les mauvais traitements de la famille ou des patrons «éducateurs » en étaient généralement la cause. Les récits reviennent ainsi sur l'argent prêté ou volé pour financer le voyage en bus vers la capitale, ville de tous les espoirs. La recommandation d'une señora rencontrée dans le voisinage, ou encore dans ce même bus, devenait le sésame pour entrer et s'employer dans une famille comme bonne interna. Margarita, née dans les années 1950, a ainsi quitté la maison familiale lorsqu'elle avait 13 ans. Elle habitait à Pereira avec sa mère et ses frères et soeurs, et s'est enfuie seule vers Cali pour travailler comme petite bonne. Elle a ensuite pu intégrer une usine agroalimentaire. Mais, alors qu'elle ne trouvait plus d'emploi, des amies lui ont proposé de fréquenter les maisons closes. Elle a 
continué ainsi, entre prostitution et service domestique, à Bogotá ${ }^{14}$. De la même manière, Angelica, actuellement employée domestique dans la capitale colombienne, a fui très jeune la maison de sa mère : «A l'âge de douze ou treize ans [j'ai décidé de partir], je suis allée voir mon grand père et je lui ai demandé de l'argent. (...) Avec trois pesos je suis allée à Popayan. Le monsieur... c'était un homme connu dans le village. C'est pour ça qu'il m'a amenée et il m'a recommandée dans une maison. »

La fuite ne peut cependant être comprise comme une règle. Toutefois, elle témoigne d'une certaine volonté d'indépendance des petites filles à une époque donnée en Colombie. Le grand nombre d'enfants dans la fratrie, les familles recomposées ainsi que le problème des enfants non reconnus peuvent expliquer, en partie, ces départs volontaires. Par ailleurs, il est important de souligner ici la référence à la «recommandation», élément que nous retrouverons ensuite de manière répétée dans les conversations et observations de terrain, tant pour les migrations que l'on pourrait qualifier de « traditionnelles » que pour celles qui ont trait au déplacement forcé.

\section{Exode, déstructurations familiales et stratégies de survie (2000)}

Le profil et le contexte migratoire des femmes déplacées par les violences dans les années 2000 et qui trouveront du travail à Bogotá comme employées domestiques, sont tout autres. En effet, les raisons du déplacement, tout d'abord, sont bien différentes. Loin du placement familial, à visée éducative ou économique, ou encore de la migration de jeunes femmes seules, pleines d'espoir d'une vie meilleure, les migrations forcées sont synonymes de pertes, d'arrachement, de deuil et de traumatismes. A l'origine du déplacement, il y a, dans $82 \%$ des cas ${ }^{15}$ [FAMIG-CODHES, 2007, p. 59], des menaces directes sur la vie des personnes, liées à plusieurs facteurs. L'accusation de collaboration avec l'armée ou avec un groupe armé ennemi, la résistance à donner la vacuna ${ }^{16}$ et le recrutement forcé du chef de famille sont autant de raisons d'être menacés et dans l'obligation de quitter le territoire précipitamment [FAMIG-CODHES, 2007, p. 59]. De cette manière, Gisela ${ }^{17}, 35$ ans, a fui soudainement le Santander quand son mari a été contraint de rejoindre un groupe armé, la laissant seule avec ses trois enfants, dans une situation de profonde détresse psychologique et économique. Par ailleurs, les départs précipités peuvent être également la conséquence de l'assassinat ou de la disparition d'un ou plusieurs membres de la famille. Claudia, qui a aujourd'hui une trentaine d'années, a ainsi perdu ses trois frères et son père, tués successivement dans le Chocó. Elle vit actuellement à Bogotá avec ses deux fils et travaille comme domestique externa. 
Tous les acteurs armés (les guérillas, les groupes paramilitaires, et dans une moindre mesure l'armée) sont impliqués dans les déplacements forcés de population. L'ensemble du groupe familial étant menacé, les survivants migreront vers la ville la plus proche ou la métropole (grands-parents et petits enfants, mère veuve et enfants, etc.). Il s'agit alors d'une migration familiale. Patricia, 34 ans, est ainsi partie dans l'urgence du Huila avec sa mère et ses deux enfants, à la suite de menaces de mort. Elle raconte : «Je suis rentrée cette nuit là et ils sont arrivés à 10 heures du soir ou quelque chose comme ça. J'ai pleuré toute la nuit, mais ensuite j'ai commencé à me demander : qu'est ce que j'emporte? J'ai pris mes papiers parce que je me suis dit: "Je ne reste pas ici! Non je ne reste pas ici! » Pour mes enfants, il vaut mieux avoir une mère vivante que morte. [...] Le premier bus partait à 5 heures du matin. Alors je suis allée à la station de bus avec mes enfants et ma maman et j'ai commencé à appeler toute la famille. »

L'enjeu du déplacement forcé, véritable tactique de guerre de la part des acteurs en présence [Osorio Pérez, 2002, p. VI], repose sur le contrôle territorial. De cette manière les petits propriétaires terriens, paysans pour la majorité, seront les premiers visés. Ils perdront, à travers le déplacement, tous leurs biens, laissés aux mains du groupe responsable du déplacement. Donny Merteens qualifie les femmes déplacées de victimes à trois niveaux : celui du traumatisme produit par les violences politiques tout d'abord (l'assassinat du conjoint ou d'autres membres de la famille, l'incendie de sa maison ou encore le viol) ; celui de la perte des moyens de subsistance (maison, animaux, cultures); et enfin celui du déracinement social et émotionnel lié au départ de zones rurales pour se diriger vers de grands centres urbains [2000, p. 382]. Selon la même auteur : «La destruction [...] va bien au delà de ses effets matériels : il s'agit d'une perte d'identité comme citoyens et sujets politiques, et d'une rupture du tissu social, à la fois familial et de la communauté, qui produit la sensation d'être complètement à la dérive. $\gg^{18}$ [Merteens, 2000, p. 382].

Dans des enquêtes réalisées à Cali, une ville du sud-ouest de la Colombie, Michel Agier et Odile Hoffmann mettaient en avant la forte déstructuration familiale des foyers de déplacés. Cette déstructuration est due à la fois aux causes même du déplacement forcé (quand le conjoint est assassiné par exemple) et aux conséquences de l'arrivée en ville qui implique souvent des conflits, séparations et dispersion des enfants [1999, p. 125]. Par ailleurs, la prééminence de femmes chefs de famille est maintenant bien connue. Cette plus grande représentation des femmes à la tête des foyers déplacés est liée selon le rapport de l'ONG CODHES et de la Fondation d'Aide aux Migrants 
à «l'augmentation des actions armées contre la population civile, où les principales victimes des rétentions, recrutements forcés et disparitions sont les hommes ${ }^{19}$ [2007, p. 66]. Mais, ces femmes peuvent également avoir pris la responsabilité du foyer à la suite d'une séparation sur le lieu de l'exil, engendrée par les tensions, la peur ou les difficultés de la survie quotidienne. Enfin, cette responsabilité féminine a parfois pour cadre une union stable. Elle résulte alors de la plus grande facilité des femme déplacées à résoudre les problèmes de subsistance de la famille [Merteens, 2000, p. 383].

La détérioration du niveau de vie des familles déplacées après la migration est très nette. Les chefs de famille ont généralement de grandes difficultés à trouver un travail et à se reconvertir dans des activités de type urbain. En effet, il s'agit souvent de personnes issues du milieu rural et pour lesquelles l'agriculture et l'élevage constituaient les principales ressources. Le taux de chômage atteint ainsi 37\% dans cette population [FAMIG-CODHES, 2007, p. 76]. Par ailleurs, lorsque les déplacés arrivent à trouver un emploi il s'agit d'activités très précaires et mal payées comme la vente ambulante de fruits, légumes, bonbons ou autres aliments dans la rue et les bus, le reciclaje ${ }^{20}$, la manutention dans des marchés en gros ou les activités domestiques à la journée. Leurs revenus moyens ne dépassent pas les 100000 pesos mensuels (environ 30 euros) pour 52\% d'entre eux [FAMIG-CODHES, 2007, p. 77], c'est-à-dire un peu moins du tiers du revenu minimum. Enfin, la difficulté à se stabiliser financièrement ainsi que la très grande précarité sont des soucis permanents. Dans ce contexte, il faut cependant noter, à la suite de Donny Meertens, que les femmes ont tendance à rechercher plus rapidement que les hommes des stratégies de survie pour pouvoir nourrir leur famille et en particulier leurs enfants. Par surcroît, elles auront plus d'opportunités de travail que leurs conjoints, par leur savoir-faire ménager qu'elles peuvent aisément reconvertir dans la ville, à travers le service domestique rémunéré notamment [2000, p. 385]. Toutefois, ces solutions de «débrouille» restent très aléatoires et n'empêchent en rien la forte paupérisation décrite précédemment. En effet, dans les quartiers les plus pauvres de Bogotá et de Soacha, des formes de trocs apparaissent, en raison du surplus de main d'oeuvre dans le service domestique : en lieu et place d'un salaire, les femmes reçoivent, pour la garde d'un enfant ou le ménage à la journée, un peu de nourriture ou un repas. Si elles sont payées, elles ne perçoivent pas plus de 5000 pesos à la journée (1,50 euros) $)^{21}$ [FAMIG-CODHES, 2007, p. 77].

Ainsi, les parcours migratoires et les caractéristiques biographiques des femmes déplacées sont-ils particulièrement distincts de ceux des petites filles et jeunes femmes décrits précédemment 
et qui ont eu cours dans les années 1960-1970. L'insertion des déplacés comme employés domestiques, dans des familles employeuses, si elle dépend parfois de dynamiques familiales ou de proximitée ${ }^{2}$, est le plus souvent liée à la mise en place de réseaux de confiance et de solidarité. Comme le montre Donny Meertens [2000], les femmes déplacées savent faire appel à l'entraide, souvent à d'autres femmes, pour trouver un emploi ou des ressources (nourriture, habits, etc.). Elles sont en cela plus «débrouillardes » que les hommes, plus habituées qu'eux aux canaux informels [2000, p. 383]. Une rencontre furtive avec des voisins, des membres d'associations de déplacés ou encore des paisanos (personnes originaires de la même région) leur permettra d'être recommandées dans une maison pour travailler quelques jours à la semaine comme employées de maison. C'est grâce à cette recommandation, et moins au statut de membre de la famille ou de personne connue, que les personnes déplacées pourront rentrer dans les familles d'employeurs, en dépit de la forte discrimination dont elles sont l'objet par ailleurs. Comment fonctionne cette « recommandation»? Pourquoi et en quoi permet-elle de faire entrer des personnes étrangères dans le foyer ? Quelles caractéristiques autorisent le dépassement des différences si marquées entre migrations des années 1960-1970 et déplacements forcés dans les années 2000 ?

\section{La « recommandation » ou comment domestiquer l'arrivée de l'étrange(r) dans le foyer}

$\mathrm{La}$ « recommandation » ou « recomendación » («la señora que me recomendó », « la niña que recomendé $»^{23}$ ) peut prendre plusieurs formes - que ce soit pour les migrations des années 1960-1970 comme pour le phénomène de déplacement forcé des années 2000. Dans son sens le plus courant, elle se rapporte au fait qu'un employeur « recommande » une de ses anciennes employées domestiques, souvent de manière orale, à un ami ou connaissance. Mais, cette recommandation désigne plus largement toute personne qui s'engage pour une employée vis-à-vis de ses potentiels employeurs. La recommandation est alors d'une grande importance car elle assure de la fiabilité et du degré de confiance qu'on peut avoir envers cette domestique : elle ne va pas voler, ni avoir de mauvaises «manies », puisque telle personne, que je connais, me l'a recommandée.

Notre hypothèse est que ce phénomène suit d'abord le fil des relations familiales : l'on recommande avant tout sa soeur ou encore sa cousine, ou tout autre membre de la famille. Il peut s'agir, comme il était courant dans les années 1960-1970, d'oncles ou de tantes qui viennent chercher une petite nièce au village car une «señora » a demandé une personne de confiance pour 
travailler chez elle. Une employée domestique peut également, une fois qu'elle s'est familiarisée avec la ville et qu'elle a consolidé son réseau de connaissances, « recommander » une soeur ou une cousine chez d'anciens employeurs, phénomènes que l'on rencontre encore aujourd'hui.

Notre recherche a montré que les employées domestiques vont, très souvent, jouer avec le schème familial de la recommandation : elles diront à des employeurs de leur connaissance qu'une certaine jeune fille est leur cousine ou leur nièce pour lui permettre de rentrer dans la maison plus facilement. Dans les années 1960, Sofia, soeur aînée d'Andrea, a ainsi été placée comme petite bonne chez des connaissances, après avoir été abandonnée par ses parents. Après l'adoption d'Andrea, les parents honteux étaient partis précipitamment en laissant leurs trois enfants les plus jeunes au village. Sofia a commencé à travailler dans une ferme des Llanos, d'où elle s'est enfuie pour revenir en Boyacá, sa région natale. Comme ses nouveaux patrons ne la payaient pas, ses amies ont été voir d'autres employeurs : «Elles ont dit que j'étais de leur famille, pour qu'ils ne me traitent pas mal et pour qu'ils me paient. Elles leur ont expliqué : "J'ai une cousine qui peut travailler pour vous, et elle ne va pas vous voler ni rien. »

Cette recommandation familiale, réelle ou feinte, interroge singulièrement les mécanismes d'entrée dans une famille étrangère. Pour domestiquer la présence intruse, il faut recréer des liens familiaux. En effet, en s'assurant que son employée n'est pas une inconnue, mais la soeur, la cousine ou la filleule de telle personne qu'ils connaissent, et en qui ils ont pleine confiance, les employeurs étendent la famille au-delà de ses limites formelles.

Du côté des patrons, le schème familial prédomine également, aujourd'hui comme hier. On va se «prêter » très fréquemment les employées domestiques, le plus souvent dans le strict cercle familial. Une employée de maison travaillera ainsi simultanément chez des soeurs ou encore des parents et enfants. Le privilège d'avoir une employée de confiance semble suivre le fil des relations de parenté, comme si, une fois entrée dans une maison, une employée qui a fait ses preuves, pouvait alors librement circuler dans les différents foyers de la famille étendue.

Ainsi, Angelica a-t-elle commencé à travailler interna chez un couple habitant un quartier de classe moyenne de Bogotá. Lorsqu'ils sont partis vivre à Mesitas, pour raisons de santé, elle a été faire le ménage chez leurs enfants. Puis, une voisine, belle-mère du fils de ses anciens patrons, a fait 
appel à ses services. Estela (35 ans) s'est employée pour sa part dans une famille à Suba (quartier nord de Bogotá). «Et puis elle m'a envoyée chez sa fille pour que j'aille lui faire le ménage », raconte-elle. Enfin, Sofia travaille dans une maison du lundi au samedi. Mais, le mercredi est réservé au ménage et aux lessives chez la soeur de sa patronne.

Cette recommandation «familiale », plus courante dans les années 1960-1970, peut être remplacée par une recommandation « amicale », comme dans le cas des personnes déplacées. Ceux qui recommandent sont les employeurs ou de potentiels employeurs, mais aussi des employées domestiques. Une employée de confiance sera ainsi recommandée à des amis. De même, les domestiques vont se recommander entre elles. Il s'agira de personnes connues de longue date, mais également de jeunes femmes que l'on désire aider et que l'on a simplement rencontrées dans la rue, dans le bus, à la boulangerie, ou encore dans le même immeuble.

Lors de notre travail de terrain dans une résidence fermée du centre ville de Bogotá, nous avons souvent vu Sofia se lier d'amitié avec les autres employées domestiques de l'immeuble. Lorsqu'elles étaient jeunes et inexpérimentées, elle les aidait à trouver du travail. Claudia, arrivée comme déplacée à Bogotá il y a une dizaine d'années, nous confie également: "J'ai trouvé du travail par recommandation. Et ça peut être comme ça: on monte dans le bus, et on se fait une petite amie. On lui donne son numéro de téléphone et voilà! Ou alors dans l'immeuble où on travaille il y a toujours une autre employée domestique. Alors on parle avec elle. Des fois, pas toujours. Et elles disent «Ah je te recommande! Quand tu as besoin, tu me dis. »» Ainsi, les domestiques jouent-elles avec les codes d'entrée et les conditions posées par les patrons. Elles insisteront pour que leur nouvelle amie dise à ses futurs patrons qu'elles se connaissent de longue date, alors que ce n'est parfois pas le cas. Marta qui a un peu plus d'une trentaine d'années, et est déplacée dans la capitale depuis deux ans, raconte comment elle a pu trouver du travail, à son arrivée à Bogotá : "Il y avait une femme là où je vis, et elle allait tous les jours dans une résidence fermée faire le ménage. Un jour, une voisine de sa patronne lui a dit qu'elle avait besoin d'une fille (...) « une fille en qui tu as confiance, qui ne va pas me voler ni rien. » (...) Et mon amie lui a dit : «Ah oui j'en connais une! » Alors elle est venue me voir : «Je lui ai dit que tu étais super! Que je te connaissais depuis je ne sais combien de mois ! Alors fais attention à ce que tu lui racontes ! „Et moi : «non, non ne t'inquiète pas. » » 
Toutefois, cette impression de rapide et facile intégration des femmes déplacées dans le monde du travail, et en particulier le service domestique, grâce à la recommandation, ne doit pas masquer la forte discrimination des migrants forcés. En effet, cette recommandation peut être très difficile à obtenir dès lors que l'on arrive sans aucun contact préalable dans la ville et que l'on est assimilé à la catégorie du «déplacé ». Dans ce contexte, les frontières de la confiance et de la méfiance se re-dessinent pour devenir particulièrement exigeantes et sources d'exclusion.

\section{Confianza et desconfianza}

Comme le précise Flor Edilma Osorio Pérez en conclusion de son doctorat, le déplacé représente «l'étranger» pour la société colombienne [2002, p. 329]. Cette catégorie entraîne avec elle tout un lot de stigmates [2002, p. 308]. La méfiance (desconfianza) de la société nationale, et de la population urbaine en particulier, envers les personnes déplacées est de plusieurs ordres. A la fois politique et sociale, elle implique des formes de rejet qui sont liées à la représentation du migrant forcé comme allié d'un camp ou d'un autre ou comme délinquant [Meertens, 1999, p. 416]. D’une part, puisqu'ils sont soupçonnés d'appartenir à un groupe armé, ce «ne sont pas des personnes en qui l'on peut avoir confiance » (confianza) [Nubía Bello et Mosquera, 1999, p. 464]. Du statut de victimes, les personnes déplacées glissent alors insensiblement vers celui de responsables du déplacement et de la tragédie vécue. Ainsi, une personne interviewée par Martha Nubía Bello et Claudia Mosquera dans un quartier populaire de Bogotá affirmait : « « Ce mois-ci beaucoup de gens sont arrivés dans le quartier, et on peut voir à leur tête qu'ils sont de la campagne. On ne peut pas en être sûrs, mais on se doute bien que ce sont des déplacés [...] Et personne ne va les embêter car ces gens là, si ce ne sont pas des guérilleros, ce sont des paramilitaires. Ça tu peux en être certain! » $»^{24}$ [1999, p. 464] La formule convenue, souvent entendue, « si ils sont déplacés c'est bien pour quelque chose », entraîne selon Flor Edilma Osorio Pérez, une légitimation silencieuse des actions violentes et justifie, par ailleurs, des «mécanismes d'impunité, d'indifférence et de consolidation de la justice privée » [2002, p. 310], si spécifiques à la situation colombienne contemporaine. D'autre part, la très forte paupérisation de cette population produit une stigmatisation sociale d'un ordre bien particulier: celle du délinquant potentiel. Enfin, la desconfianza peut également résulter d'une concurrence pour les ressources publiques (sous formes d'aides sociales), de la représentation du déplacé comme mendiant et, parfois, imposteur ${ }^{25}$ [Osorio Pérez, 2002, p. 308]. 
Dans le cadre du service domestique, cette méfiance envers les populations déplacées peut avoir un impact sur les mécanismes de recommandation décrits plus haut, mais également sur l'acceptation ou non du migrant forcé au sein même du foyer. La sensation d'insécurité, la volonté de protéger son espace intime et son environnement familial peuvent alors freiner l'emploi d'une personne déplacée comme domestique. Ainsi, l'expérience de Lucía montre-t-elle tous les obstacles auxquels sont confrontées ces femmes. Lucía a une trentaine d'années. Elle a fui le Meta avec ses deux fils et sa fille, à la suite de l'assassinat de son mari par un groupe armé il y a un an et demi. Lorsque nous l'avons rencontrée dans une association d'aide aux déplacées, elle était à la recherche d'un emploi. Sa situation de détresse était d'autant plus grande que son ancienne employeuse chez qui elle faisait le ménage trois fois par semaine était partie il y a peu à Medellín. Elle raconte alors toute la difficulté à trouver du travail dans cette ville profondément hostile. En effet, elle ne connaît personne qui puisse la recommander, puisqu'elle est partie de manière spontanée et dans l'urgence, sans contact préalable. Par ailleurs, elle a avec elle ses trois enfants en bas âge et ne peut donc pas s'employer comme bonne interna ${ }^{26}$. Enfin, elle ne dispose d'aucun réseau familial ou amical, dans une ville où la méfiance règne. Bogotá est, pour elle, fermée à toute opportunité : «Ici personne comme ça du jour au lendemain ne va te donner la possibilité de travailler. Personne ne t'ouvre les portes et te dit : «Viens! Viens, je vais te donner du travail ». Personne ne le fait». Ce à quoi elle ajoute : «Ici, il faut cacher qu'on est déplacés. (...) Car les gens pensent qu'on va les voler, ou qu'on va leur faire je ne sais quoi. Les gens sont très méfiants (« desconfiados »). Avec nous les déplacés, les gens sont très méfiants. » Et pourtant, « cacher » semble être particulièrement difficile. Alors que les recommandations finalement obtenues proviennent pour l'essentiel du voisinage, ici «les gens regardent. Ils sont malins. Et ils se demandent : "Ceux là d'où ils viennent ? Pourquoi ils sont là ? »Ils cherchent, ils cherchent et finalement : «Ah oui, ce sont des déplacés! ».

Pour Lucía, c'est la porte des maisonnées qui sera le plus difficile à franchir : «Il se peut qu'on vous offre du travail dans d'autres secteurs, pour vendre quelque chose (...) mais dans les maisons c'est très compliqué de pouvoir rentrer. » Ce métier si difficile à obtenir sera le plus recherché car il permet un salaire fixe et une certaine stabilité de l'emploi, précieux dès lors que l'on a des enfants et un loyer à payer. Tout l'enjeu sera de trouver des contacts qui puissent attester de sa fiabilité : «C'est un problème, il faut que quelqu'un te recommande. Qui dise: «bon je la recommande car elle est avec moi, elle a travaillé... Enfin, c'est-à-dire qu'il faut comme un crochet 
pour pouvoir rentrer et travailler comme ça por días. »

Ce « crochet », Pilar n'en a pas eu besoin, elle qui est arrivée à Bogotá à la fin des années 1970. Les personnes rencontrées dans le voisinage de son lieu de travail venaient souvent lui demander si elle pouvait travailler pour eux. Lucía, pour sa part, a bien été recommandée par une de ses premières patronnes qui vivait dans un quartier chic de Bogotá. Mais, alors que cette patronne tente de la présenter à une de ses amies, celle-ci s'exclame «Ah non alors! Moi des personnes déplacées je n'en veux pas! A ces gens-là, je ne leur donne pas d'emploi! Qui sait ce qu'ils auront fait! Et si on les a sortis de là bas, c'est bien pour quelque chose... Non, ma fille, je suis désolée, tu peux me la recommander et tout, elle peut travailler avec toi, mais non madame! »

Cependant, des réactions de solidarité dans le voisinage, les associations de déplacés, les Églises, vont permettre de dépasser cette stigmatisation première. Ainsi Lucía a-t-elle trouvé son travail avec son ancienne employeuse grâce à une femme de son quartier. Elle attendait son tour pour acheter du pain et se plaignait de sa délicate situation financière à son fils, lorsqu'une femme derrière elle l'a abordée en lui disant qu'elle pouvait l'aider. «Vous avez une expérience comme employée domestique? », lui a-t-elle demandé. Et effectivement, Lucía avait travaillé à Villavicencio comme bonne interna, il y a une dizaine d'années. Elle avait vingt ans, était célibataire et sans enfants. Par la suite, elle est repartie avec son mari dans la campagne environnante pour s'employer dans une ferme comme administratrice ${ }^{27}$.

A son arrivée à Bogotá, Lucía a fait du reciclaje pendant trois mois pour subvenir à ses besoins et à ceux de ses enfants. Puis, elle a pu intégrer un programme d'aide aux déplacés, formation de quelques semaines dans un jardin botanique. Elle a ainsi rencontré une employée du programme. Cette personne l'a recommandée auprès d'une future patronne chez qui elle a été faire le ménage pendant trois mois et demi avant son départ aux Etats Unis. Lors de leur première entrevue, cette employeuse lui a demandé : «Vous êtes déplacée ?», «J'ai répondu que oui. Je pensais qu'à cause de cela elle n'allait pas me donner le travail. Mais elle a dit : "Moi ça m'intéresse. Parce que parfois ce sont les personnes que l'on discrimine le plus dont a le plus besoin!»

Les sociologues et anthropologues, spécialistes des migrations forcées, ont déjà noté de nombreuses fois la présence d'une solidarité spécifique au déplacement forcé. Si certains 
stigmatisent cette population, d'autres au contraire les considèrent comme des victimes innocentes qui nécessitent l'aide d'autrui. Martha Nubía Bello et Claudia Mosquera ont montré comment ces réseaux d'aides se mettent en place dans les quartiers, légitimés notamment par la foi et le culte évangéliques, très présents dans les zones populaires [1999, p. 464]. Toutefois, la question spécifique des rapports patrons/employées a été peu traitée. Dans le cas du service domestique, elle est pourtant d'un grand intérêt. Il est maintenant bien connu que la domesticité permet de mettre en contact des personnes de différentes classes sociales [Chaney et Castro, 1993 (1989)]. Plus récemment, les études sociologiques et anthropologiques sur le service domestique se sont intéressées à la rencontre, à travers cet emploi, de patrons et employés de diverses origines ethniques [Barrig, 2001]. Mais, le fait qu'il induise également la rencontre de deux parties de la société colombienne totalement étrangères l'une à l'autre - les victimes du conflit armé et ceux qui en sont protégés - est resté dans l'ombre. Michel Agier [2002] analyse, dans un ouvrage qu'il consacre aux réfugiés, l'occupation, par des déplacés colombiens, du siège de la Croix Rouge internationale dans une zone huppée de Bogotá. Il explique ainsi comment, pendant ces quelques mois, deux pans d'humanités se sont regardées, « hagardes », alors qu'elles ne se voient jamais : les déplacés et les habitants privilégiés des quartiers nord - la zona rosa, lieu de l'occupation [2002, p. 65-66]. Cette absence de rencontre est le fait d'un confinement des personnes déplacées dans les zones périphériques et miséreuses de la capitale colombienne. Mais, la peur de la souillure et de la contamination de personnes qui ont vécu, de trop près, les massacres, exactions, trahisons et complicités forcées d'une guerre niée [Agier, 2002, p. 59], jouent sans doute un grand rôle dans cette ignorance. Pourtant, comme le montre l'histoire de Lucía, tous les patrons, n'ont pas la même réaction d'évitement des personnes déplacées.

Le témoignage de Marta nous permet d'aller plus loin encore dans cette analyse des modalités d'entrée des domestiques déplacées dans les familles employeuses et la mise en place de rapports privilégiés. Marta est originaire du Caqueta, au sud du pays. Son père a été recruté de force par un groupe armé il y a une quinzaine d'années. Depuis, elle est sans nouvelles de lui. Quant à son frère, il s'est enfui du village pour fuir les enrôlements. La mère de Marta est ainsi la seule famille proche qui lui reste, avec ses deux fils. Dans sa région natale, elle travaillait dans une boutique de confection et avait une petite maison coquette. Mais, lorsqu'on a menacé de lui enlever ses enfants pour en faire des soldats d'armées illégales, elle a décidé de s'enfuir et est allée à Bogotá chez des personnes qu'elle avait connues plusieurs années auparavant. Elle est maintenant logée dans une 
petite chambre de Ciudad Bolivar, un des quartiers les plus miséreux et violents de la capitale. Alors qu'elle jouissait d'un statut social correct dans sa ville natale, le gaz et l'électricité sont coupés quand nous lui rendons visite. Elle ne nous offre rien à boire, un peu peinée. Elle raconte : «la majeure partie de ce que tu vois ici m'a été offerte. (...) La seule chose que j'ai achetée c'est le téléviseur». Ces cadeaux, elle les a reçus des voisins, amis ou fondations d'aide aux déplacés. Les familles chez lesquelles elle va faire le ménage quelques jours à la semaine lui ont également donné quelques biens, nourriture, habits. Une femme en particulier qui « vit bien au nord, nord, nord... dans la rue 100 ou $200^{28}$ », chez qui elle a été recommandée par une voisine, l'a beaucoup aidée : «Au début je me suis tue. Parce que vous savez, les gens disent que les déplacés sont des voleurs, que le déplacé est un délinquant... mais après elle m'a demandé d'où j'étais, alors je lui ai raconté que j'étais déplacée [...] Elle s'est exclamée : "Mais pourquoi tu ne me l'as pas dit avant! [...] Et combien d'enfants tu as? "Elle m'a offert des chaussures, des petites chemises. Elle me disait : "J'ai parlé avec une amie qui a un enfant, elle va nous donner des affaires! »»

Marta travaille également comme employée de maison chez trois soeurs, qu'elle a connues grâce à des amis de sa région natale. Elles savent, bien évidemment, qu'elle est déplacée et veulent lui rendre service. Leur aide passe généralement par une prise en charge des enfants. Elles appellent Marta pour qu'elle vienne faire le ménage chez chacune d'elle. Marta reste à la maison, lave les sols et les murs, pendant que ses fils vont avec ses patronnes, en voiture, et ne reviennent que le soir avec des glaces et des ballons. Parfois, ils restent dans une des maisons et jouent avec les enfants des employeurs : «tu verrais avec la petite fille ils font de ces châteaux de draps et de couvertures !». Et d'ajouter : «Alors c'est comme si ils étaient... ils ne font jamais cette différence (...) c'est comme si c'était la famille ».

Dans les parcours des femmes qui ont commencé à travailler comme domestiques dans les années 1960-1970, nous avons souvent rencontré ce traitement privilégié des enfants. Des pratiques de parrainage des enfants d'employées par les patrons sont fréquentes. Par ailleurs, les processus de «pseudo-intégration familiale » dans les rapports employeurs/domestiques ont été mis en avant par la littérature sur la domesticité [Anderfuhren, 1999, p. 54]. Il est ainsi à la fois tout naturel - car ce sont des pratiques anciennes -, et étonnant - en raison du stigmate du déplacé -, de retrouver cette « intégration familiale », le temps d'un goûter ou d'une après-midi de loisir. Au-delà de la figure du déplacé-mendiant, classe dangereuse et/ou victime, des liens se nouent, qui ramènent au statut 
traditionnel de l'employée domestique en Colombie.

\section{Conclusion}

La comparaison entre les parcours migratoires et l'insertion dans les familles, comme employées domestiques, des petites filles ou jeunes femmes dans les années 1960-1970 et des femmes déplacées dans les années 2000 a mis en évidence la « recommandation » comme mode de «domestication » de la présence étrangère au sein du foyer colombien. Cette recommandation peut être particulièrement difficile à obtenir pour les femmes déplacées en raison du stigmate du déplacement forcé et du peu de contacts dont elles disposent. Toutefois, très vite le voisinage, les Églises, les associations d'aides aux déplacés se mobilisent pour créer des réseaux de solidarité qui permettent d'être « recommandée » dans des familles connues ou amies. Du côté des employeurs, si les réactions d'évitement et de stigmatisation sont premières, une forme d'aide apparaîtra également, sous forme de dons d'habits ou de nourriture. Enfin, la «pseudo-intégration familiale », ou « fiction familiale » [Bernardo, citée par Weber, 2005, p. 161], si typique de la domesticité partout dans le monde, se fait ici solidarité et on ne peut dénier son importance pour des femmes qui ont, souvent, perdu une partie de leur famille. Une analyse des liens particuliers qui se nouent entre famille employeuse et famille des employées domestiques en Colombie serait sans doute nécessaire pour comprendre la permanence d'un tel phénomène. 


\section{Méthodes et terrain}

nationale ECOS-Nord intitulé «Histoires de vie, histoires de femmes. L'identité féminine colombienne des années 1980 à nos jours » et par une aide au terrain de l'Ecole des Hautes Etudes en Sciences Sociales. L'observation ethnographique a guidé ma première appréhension du sujet puisque j'ai vécu pendant cinq mois dans une résidence fermée successivement chez deux familles qui employaient chacune une travailleuse domestique. J'ai ainsi pu saisir toute l'ambiguïté des rapports employeurs-employées, entre simili-amitié et rancoeurs partagées. J'ai quitté le logement d'une de ces familles pour ne plus avoir de rapports avec la patronne-employeuse et ainsi pouvoir établir une relation sereine avec l'employée domestique, qui m'avait confié de grands pans de sa vie lorsque nous nous retrouvions seule à seule dans la maison. Je me suis alors aperçue de la très grande difficulté à travailler sur ce sujet depuis une position d'employeuse, ou en entretenant des relations amicales avec les patrons de domestiques. En effet, chaque fois qu'une amie employeuse me proposait de faire un entretien avec son employée de maison, il tournait court, ou tout simplement n'avait pas lieu. En Colombie, pourtant, les premières enquêtes sur les travailleuses domestiques ont été effectuées par des universitaires féministes colombiennes [León, 1987 ; Chaney et Castro, 1989] - qui étaient par ailleurs des patronnes. Ces recherches se faisaient en collaboration avec des syndicats de travailleuses domestiques et avaient pour but de transformer la société colombienne en vue d'un meilleur respect du droit du travail. La position réflexive était privilégiée, ainsi qu'une forte remise en question des relations de pouvoir entre femmes de classes sociales distinctes. A l'extrême inverse de cette posture de «patronne réflexive », Judith Rollins a choisi de prendre, pour un temps, la place d'une employée domestique en se faisant engager comme femme de ménage un jour par semaine chez différents employeurs [1990, p. 64]. Cette expérience lui a permis de mettre au jour un certain nombre de caractéristiques physiques du travail domestique : la dureté des tâches, la solitude et la monotonie. Elle a également été le socle d'une critique radicale de l'oppression de race et de classe à l'oeuvre dans ce type de travail. Entre ces deux positions, impliquées de manière toute particulière, j'ai choisi de me situer dans un entredeux de neutralité. L'expérience, forcée et non provoquée, de relations patrons-employées avec les domestiques des familles qui m'accueillaient m'a sensibilisée à une vision non manichéenne de ces rapports. Etre patron est un art complexe, et les abus existent de part et d'autre. Toutefois la posture de patronne a ses limites et ne permet parfois pas d'entrer en profondeur dans la réalité du quotidien des domestiques : leur vie de famille, leurs attentes et leurs espoirs. De cette manière, j'ai choisi d'aller vivre seule, sans engager de bonne, et d'approfondir des relations d'amitiés avec cinq familles d'employées domestiques. J'ai également effectué une trentaine d'entretiens ouverts avec des employées de maison. Pour cela j'ai travaillé en collaboration avec une école maternelle d'un quartier populaire de Bogotá et un programme social de la Mairie. J'ai alors rencontré des difficultés pour trouver un cadre et un moment adéquats pour faire ces entretiens, les employées domestiques interviewées étant particulièrement occupées par leur vie de famille ou encore par le programme de réinsertion sociale de la Mairie. Enfin, j'ai utilisé diverses sources littéraires et bibliographiques issues du Centre de Genre de l'Université Nationale de Colombie, des bibliothèques centrales de l'Université des Andes et de l'Université Javeriana, de la bibliothèque publique Luis Angel Arango, de l'Institut Colombien d'Anthropologie et d'Histoire et du centre de documentation de l'O.N.G. Save The Children. En ce qui concerne spécifiquement le déplacement forcé, j'ai travaillé en collaboration avec deux institutions gouvernementales et une fondation religieuse. Par leur biais, j'ai pu rencontrer une dizaine de femmes déplacées de tous âges et de toutes origines ethniques. Puis, j'ai effectué des entretiens formels ouverts de deux heures avec quatre d'entre elles. Il me faut préciser, pour conclure, que le nombre de femmes déplacées employées comme domestiques dans la capitale colombienne est très difficile à évaluer. En effet, la dernière étude, analyse de données statistiques, sur le travail domestique à Bogotá ne prend pas en compte le déplacement forcé pour expliciter et classer ses résultats [Cárdenas et Harker, 2006]. 


\section{Bibliographie}

Agier Michel, 2002, Aux bords du monde, les réfugiés, Paris, Flammarion.

Agier Michel, Hoffmann Odile, 1999, « Pérdida de lugar, despojo y urbanización. Un estudio sobre los desplazados en Colombia » in Cubides Fernando, Domínguez Camilo (dir.), Desplazados, migraciones internas y reestrucuraciones territoriales, Bogotá, Universidad Nacional de Colombia, pp. 104-126.

Anderfuhren Marie, 1999, L'employée domestique à Recife (Brésil). Entre subordination et recherche d'autonomie, Paris, Thèse de doctorat, Université de Paris I - Panthéon Sorbonne, Institut d'Etude du développement économique et social.

Barreto Juanita Gama, 2001, Trabajo doméstico infantil y juvenil en hogares ajenos : de la formulación de los derechos a su aplicación. Cuatro estudios locales en Colombia, Bogotá, UNICEF et Save The Children-UK.

Barrig Maruja, 2001, El mundo al revés : imágenes de la mujer indígena, Buenos Aires, Colección Becas de Investigación CLASCO-ASDI.

Cárdenas Mauricio S., Harker Arturo R., 2006, Determinantes del empleo y de los ingresos del servicio doméstico en Bogotá, Bogotá, FEDESAROLLO.

Cardona Gutierrez Ramiro (dir.), 1970, Las migraciones internas, Bogotá, Editorial Andes.

Chaney Elsa M., Castro Mary García, 1993 (1989), Muchacha, cachifa, criada, empleada, empregadinha, sirvienta y...más nada. Trabajadoras del hogar en América Latina y el Caribe, Apartado, Editorial Nueva Sociedad.

Fierro Marco, 1973, Algunos problemas relacionados con la migración interna en Colombia, Bogotá, Centro de Estudios sobre Desarrollo Económico, Universidad de los Andes, Facultad de Economía, nº 003 . 
Fundación de atención al migrante - FAMIG, Consultoría para los derechos humanos y el desplazamiento - CODHES, 2007, Gota a Gota. Desplazamiento forzado en Bogotá y Soacha, Bogotá, Fundación de atención al migrante - FAMIG, Consultoría para los derechos humanos y el desplazamiento - CODHES, Organización internacional para las migraciones - OIM.

Hoyos Hernán, 1999 (1973), Aventuras de una sirvienta, Cali, Librería Proveta.

Jacquemin Mélanie, 2009, « « Petites nièces » et « petites bonnes » à Abidjan. Les mutations de la domesticité juvénile », in Travail, Genre et Sociétés, n²2, novembre.

Jacquemin Mélanie, 2007, Sociologie du service domestique juvénile : «Petites nièces » et «petites bonnes » à Abidjan, Paris, Thèse de doctorat, Ecole des Hautes Etudes en Sciences Sociales.

Langton Karen, 2002, Doméstico sinónimo de mujer: De donde y por qué salen las niñas que trabajan en el servicio doméstico ? El caso de San Pedro de Iguaque, Bogotá, Tesis de grado, Universidad de los Andes, Facultad de ciencias sociales, Departamento de Antropología.

León Magdalena, 1987, « Colombia : trabajo doméstico y servicio doméstico » in Schuler Margaret (dir.), Poder y derecho. Estrategias de las mujeres del tercer mundo, Washington, OEF International, pp. 333-346.

Mártinez Gómez Ciro Leonardo, 2006, Las migraciones internas en Colombia. Análisis territorial y demográfico según los censos de 1973 y 1993, Bogotá, Universidad Externado de Colombia.

Meertens Donny, 2001, «Populations déplacées en Colombie et insertion urbaine » in Annales de la recherche urbaine, $\mathrm{n}^{\circ} 91$, Décembre, pp. 118-127.

Meertens Donny, 2000, Ensayos sobre tierra, violencia y género, Bogotá, Universidad Nacional de Colombia.

Meertens Donny, 1999, «Desplazamiento forzado y género: trayectorias y estrategias de reconstrucción vital » in Cubides Fernando, Domínguez Camilo (dir.), Desplazados, migraciones 
internas y reestrucuraciones territoriales, Bogotá, Universidad Nacional de Colombia, pp. 406-455.

Nubía Bello Martha, Mosquera Claudia, 1999, « Desplazados, migrantes y excluidos : actores de las dinámicas urbanas », in Cubides Fernando, Domínguez Camilo (dir.), Desplazados, migraciones internas y reestrucuraciones territoriales, Bogotá, Universidad Nacional de Colombia, pp. 456-474.

Ojeda Parra, Teresa, 2005, Prisiones domésticas, ciudadanías restringidas. Violencia sexual a trabajadoras del hogar en Lima, Lima, Universidad Peruana Cayetano Heredia (UPCH).

Osorio Lizarazo José Antonio, 2008 (1952), El día del odio, Bogotá, Editora Aguilar Altea Taurus Alfaguara S. A.

Osorio Pérez Flor Edilma, 2003a, « Migraciones internas y recomposición territorial » in Ambiente y Desarrollo, $\mathrm{n}^{\circ} 12$, pp. 29-51.

Osorio Pérez Flor Edilma, 2003b, « Todo en común...menos el dolor, la miseria y los estigmas » in Revista Javeriana, Octubre, pp. 54-61.

Osorio Pérez Flor Edilma, 2002, Los desplazados. Entre survie et résistance, territorialités et identités en suspens, Toulouse, Thèse de Doctorat, Université de Toulouse Le Mirail, GRAL.

Pécaut Daniel, 2005 (1996), « Réflexions sur la violence en Colombie », in Françoise Héritier (dir.), De la violence I, Paris, Odile Jacob, pp. 225-271.

Pécaut Daniel, 1996/1997, «De la banalité de la violence à la terreur : le cas colombien » in Cultures et conflit, $\mathrm{n}^{\circ} 24-25$, pp. 147-179.

Pécaut Daniel, 1994, «Violence et politique: quatre éléments de réflexion à propos de la Colombie » in Cultures et conflit, $\mathrm{n}^{\circ} 13-14, \mathrm{pp} .155-166$.

Quintero de Molano Bertha, 1981, «Condiciones de vida y de trabajo de las empleadas domésticas en Bogotá » in Castro Mary García, Quintero de Molano Bertha, Jimeno Gladys, Empleo 
doméstico, sector informal, migración y movilidad ocupacional en áreas urbanas en Colombia, Bogotá, Proyecto PNUD-OIT COL/72/027 Migraciones Laborales, pp. 2-84.

Ramírez, María Himelda, 2001, «El impacto del desplazamiento forzado sobre las mujeres en Colombia », Amérique Latine Histoire et Mémoire, vol. 3.

Rollins Judith, 1990, «Entre femmes. Les domestiques et leurs patronnes », Actes de la recherche en sciences sociales, $\mathrm{n}^{\circ} 84$, pp. 63-77.

Sánchez Gonzalo, 1998, Guerre et politique en Colombie, Paris-Montréal, L'Harmattan.

Torales Porciano, 1979, La dinámica interna de los movimientos migratorios en Colombia, Bogotá, Proyecto PNUD-OIT COL 72/027, Ministerio de Trabajo y Seguridad Social SENALDE.

Vidal Dominique, 2007, Les bonnes de Rio, Emploi domestique et société démocratique au Brésil, Villeneuve d'Ascq, Presses Universitaires du Septentrion.

Weber Florence, 2005, Le sang, le nom, le quotidien. Une sociologie de la parenté pratique, La Courneuve, Editions Aux lieux d'être. 
1 Nous entendons ici par «villes intermédiaires » des villages ou petites villes de Cundinamarca ou Boyacá où les jeunes filles, qui allaient ensuite s'employer dans la capitale, étaient parfois mises au travail comme petites bonnes.

2 Le Bogotázo est le nom communément donné au 9 avril 1948, jour de l'assassinat de Jorge Eliécer Gaitán, chef de file libéral et candidat aux élections présidentielles. Cet événement a entraîné une révolte populaire qui a mis le centre ville de Bogotá à feu et à sang. Les protestations contre le gouvernement conservateur se sont ensuite étendues à l'ensemble du territoire national et ont marqué le début d'une période de guerre civile : La Violencia (1948-1953, toutefois on considère que les violences ne se sont arrêtées qu'au début des années 1960).

3 Boyacá est une région limitrophe à Bogotá qui vit principalement de l'agriculture, de la pêche et de l'artisanat. Durant la première moitié du XXème siècle, les petits paysans de Boyacá sont arrivés en masse dans la capitale colombienne pour s'employer dans le milieu de la construction ou encore du service domestique.

4 Le haut plateau cundiboyacense regroupe une zone andine qui s'étend sur les régions de Boyacá et Cundinamarca, proches de Bogotá.

5 En Colombie et dans de nombreux pays d'Amérique Latine, il existe plusieurs modalités d'emploi dans le service domestique : celui-ci peut être «interno » (l'employée reste dormir chez son employeur, et travaille du lundi au samedi - ou dimanche - sans horaires fixes) ou «por días » (la travailleuse domestique ne dort pas chez ses patrons et peut venir faire le ménage, la nourriture et/ou garder des enfants du lundi au samedi ou simplement quelques jours dans la semaine). On parle également parfois des employées externas. Ce terme désigne alors les femmes qui ne résident pas chez leurs employeurs, mais qui travaillent à plein temps.

6 Traduit par nos soins

7 La part exacte des personnes déplacées dans les migrations internes récentes est difficile à établir en raison de la nature des sources disponibles (voir encadré méthodologique).

8 Nous n'évoquerons, dans cette partie, que le cas des petites filles et jeunes filles. Des femmes pouvaient également s'insérer dans l'emploi domestique, à cette époque en Colombie, à un âge plus avancé. Dès lors leur « recrutement » se faisait généralement en fonction de dynamiques de « recommandations », le plus souvent familiales.

9 Cf. encadré méthodologique.

10 Les arepas sont des galettes de mais qui remplacent le pain dans la cuisine colombienne.

11 Karen Langton, dans son mémoire de fin d'études (tesis de grado), fait la même remarque en ce qui concerne les petites filles qui partent aujourd'hui de San Pedro de Iguaque (Boyacá) pour s'employer comme petites bonnes : «Le départ de ces petites filles, paysannes, pour travailler dans l'emploi domestique dans les grandes villes est un phénomène commun. Une grande partie de ces petites filles sont remises par leurs propres parents à des personnes connues ou même des membres de la famille. Leur travail sera alors une forme de rétribution pour l'éducation et la nourriture qui leur sera octroyée. » [2002, p. 1]

12 Propriétaires terriens

13 Il est maintenant avéré que de nombreuses petites filles et jeunes filles ont été violées par leurs patrons durant l'exercice de leur travail, quand elles étaient bonnes à demeure. Ce phénomène semble être commun à de nombreux pays d'Amérique Latine et un livre est spécifiquement dédié à cette question au Pérou [Ojeda Parra, 2005].

14 La question du lien, réel ou fantasmé, entre service domestique et prostitution est un thème délicat. Son omniprésence dès lors que l'on aborde le thème de la domesticité questionne. Le roman érotique des années 1970 , Aventuras de una sirvienta [Hoyos, 1999 (1973)], est un bon exemple du lot de fantasmes sexuels associés à la jeune paysanne, fraîche et naïve, récemment arrivée des campagnes environnantes, qui s'emploie comme bonne à demeure. Dans un tout autre registre, José Antonio Osorio Lizarazo [2008 (1952)], introduit dans son roman l'idée d'une continuité biographique entre prostitution et emploi domestique, puisque Transito, chassée de la maison de ses patrons, puis violée par un agent de police, est finalement forcée à se prostituer. Il s'agit là d'exemples purement romanesques, qui pourraient ne refléter que des stéréotypes, pourtant, lors d'un entretien, Pilar nous a confié qu'elle avait "échappé" à bien des tentations à son arrivée en ville, lorsqu'elle était adolescente et qu'elle travaillait comme domestique dans la capitale, notamment des offres qui lui auraient été faites par des amies à se prostituer. Il est difficile cependant de généraliser à partir de si peu d'exemples et le passage de la domesticité à la prostitution reste sans doute exceptionnel. Un travail spécifique sur le sujet serait néanmoins nécessaire pour plus de précisions.

15 L'enquête citée a été effectuée par la FAMIG (Fondation d'Attention aux Migrants) à Bogotá entre juillet et novembre 2006 et porte sur 480 entretiens réalisés auprès d'un échantillon représentatif de personnes déplacées entre 2001 et 2005.

16 Littéralement le «vaccin ». Il s'agit d'une extorsion d'argent, forme d'autorisation à traverser un territoire sous domination d'un groupe armé donné.

17 Pour une plus grande sécurité des personnes interviewées, certains éléments biographiques ont dû être modifiés ou omis.

18 Traduit par nos soins.

19 Traduit par nos soins.

20 Le reciclaje ou « recyclage » est une activité de «débrouille» commune dans les grandes villes qui consiste à récupérer dans la rue, souvent dans les poubelles, du carton et du plastique, par exemple, qui sont ensuite revendus à des magasins ou autres intermédiaires. Il s'agit d'une activité extrêmement dévalorisée socialement et considérée 
comme très dégradante.

21 Pour indications sur le niveau de vie en Colombie, un ticket de bus coûte actuellement entre 1000 et 1500 pesos.

22 Plusieurs auteurs ont noté que les personnes déplacées, à leur arrivée en ville, comptaient souvent sur des contacts amicaux et familiaux qui leur permettent de trouver un logement [Nubía Bello et Mosquera, 1999 ; Meertens, 1999 ].

23 «La femme qui m'a recommandé », « la jeune fille que j'ai recommandé ».

24 Traduit par nos soins.

25 En effet, les personnes déplacées sont parfois accusées de mentir sur leur statut pour pouvoir bénéficier des aides publiques qui existent pour venir en aide à cette population (ces aides publiques ne durent cependant pas plus de quelques mois).

26 Mauricio Cárdenas et Arturo Harker indiquent dans leur étude récente sur l'emploi domestique à Bogotá, qu'un tiers des employées de la capitale travaillent encore aujourd'hui comme interna (2006, p. 7). Il s'agit donc d'une modalité d'insertion dans l'emploi domestique non négligeable notamment pour les femmes récemment arrivées en ville.

27 L'on nomme administrateurs ou «mayordomos », les personnes, souvent des familles, chargées de garder les villas ou grandes fermes. Ils vivent dans une petite maison sur la propriété et sont chargés de la maintenance et de la surveillance, ainsi que de la gestion des petits paysans qui travaillent pour l'hacendado.

28 Lorsque l'on évoque le «nord » de Bogotá, on se réfère généralement aux quartiers aisés qui sont regroupés dans cette zone. 